\title{
A vicious cycle of helminth infections: Current sanitation status and practices that led to continued STH transmission in rural communities in the Philippines
}

\author{
Pauline Joy Lorenzo \\ Research Institute for Tropical Medicine \\ Charmaine Joy Berte ( $\nabla$ charmainejoyfloresberte@gmail.com ) \\ Research Institute for Tropical Medicine https://orcid.org/0000-0003-4180-9079 \\ Dazzle Kane Cortel \\ Research Institute for Tropical Medicine \\ Duane Raphael Manzanilla \\ Research Institute for Tropical Medicine \\ Christian Anthony Luna \\ Research Institute for Tropical Medicine \\ Jennifer Luchavez \\ Research Institute for Tropical Medicine \\ Winston Palasi \\ DOH: Republic of the Philippines Department of Health
}

\section{Research Article}

Keywords: open defecation, STH infection, KAP, WASH, multi-sectoral

Posted Date: September 15th, 2021

DOI: https://doi.org/10.21203/rs.3.rs-877501/v1

License: () (i) This work is licensed under a Creative Commons Attribution 4.0 International License. Read Full License 


\section{Abstract}

\section{Background}

Helminth infections among children remains a public health concern despite continued deworming activities conducted in all public schools nationwide. Access to improved sanitation facilities not only leads to continued practice of open defecation predispose children to this intestinal parasitic infection. This study aim to characterize the sanitation situation and practices among households as well as their deworming practices and associate it with the prevalence in two rural communities in San Pascual, Masbate, Philippines.

Methods

A survey of 234 randomly selected households from the two barangays was conducted followed by stool screening using KatoKatz method from 586 children and adolescents below 18 years old was done in June 2019.

Results

The survey showed that $33.28 \%$ of households do not have access to sanitary toilet and open defecation was practiced by $53.92 \%$ of surveyed households. More than half of the households (60.75\%) surveyed also reported "poso" as their source of drinking water. The survey also revealed that households have high scores in the knowledge, attitude, and practices or KAP section of the survey. The over-all cumulative prevalence rate was at $41.6 \%$ moderate to light intensity infection rate.

Conclusion

Among the variables tested, only the source of drinking water, attitude scores, and MDA participation showed association with having helminth infection among children. The study highlights the importance of advocating multi-sectoral approach in addressing persistent public health issues like the intestinal parasitic infections among children. Moreover, this study provides valuable information needed to support strategies like community-led total sanitation or CLTS as a potential strategy addressing both sanitation and public health dilemma.

\section{Introduction}

Soil-transmitted helminth or STH infection is considered as a significant public health concern in developing countries like the Philippines which have poor access to sanitation facilities (31\%) and no access to improved water sources (8\%) (WHO/UNICEF, 2012). Open defecation (OD) practice predisposes millions of people globally to STH and other intestinal parasitic diseases. OD practice can even affect 1.8 billion people who accessed drinking water that is contaminated with fecal parasites (WHO/UNICEF, 2017). A study conducted in Kenya showed that there was a higher diarrheal prevalence rate in regions where OD is reported (Njuguna, 2016). Moreover, deaths of children under five due to unsafe sanitation brings a toll of 361,000 worldwide annually (Prüss-Ustün, et al, 2014). In the Philippines, 4.5\% of households practice open defecation (PSA \& ICF, 2018).

While addressing the OD practice dilemma is done by providing hardware (toilet/latrine and sewage system) and software (practices and policies) (Albonico, Montresor, Crompton \& Savioli, 2006) components to the target areas, addressing the STH infection among children is done at schools nationwide. In the Philippines, the primary strategy for controlling the spread of these parasitic infections is by mass administration of anti-parasitic drugs (Albendazole/Mebendazole) among school-aged children. This approach serves as both preventive and treatment measure in lowering the incidence of STH infection among children. The problem with this is that reinfection is likely to happen once mass treatment is skipped or not sustained (Clements, et al, 2009; Doenhoff, Cioli, \& Utzinger, 2009; Ross, et al, 2002). In 2015, a study in Southern Leyte revealed that persistence of OD practice has led to similar continued transmission of STH among preschool aged and school aged children (Belizario et al. 2015). Since the focus of the mass drug administration of deworming drugs is on public school children, a major percentage of children remained not treated similar to the evidence found in other countries (Anderson, et al, 2013).

Achieving zero open defecation or ZOD status has been very challenging the country like the Philippines. Community-led total sanitation or CLTS was one of the strategies employed to achieve ZOD status. This participatory rural appraisal method aims to 
awaken the community about their sanitation problems and make them act upon using indigenous materials (Kar and Chambers, 2008). CLTS is now implemented in 59 countries worldwide particularly in Asia, Africa, and Latin America (Zuin, et al, 2019). A case study in Malawi showed that villages that underwent CLTS activities tend to have children showing better health stature and less likely to be stunted (Pickering, et al., 2015). While it is found effective elsewhere, there is a dearth of knowledge on how strategies like the CLTS approach works in Philippine setting; and how the intervention was received by the communities. According to the case study conducted by UNICEF East Asia and Pacific Region Office or EAPRO, the performance of the country in achieving ZOD has been hampered by challenges like lack of political will, over-reliance to international donors or nongovernment organizations, and the geographic disadvantages of the target communities (UNICEF, 2012).

The purpose of this specific paper is to characterize the prevailing knowledge, attitudes, and practices on hygiene and sanitation practices of selected households in two STH-endemic communities in San Pascual, Masbate, Philippines and determine which among those variables contribute to the persistence of STH infection among children. This paper is part of a bigger project entitled: "Ending the transmission cycle: Community-led total sanitation as an approach for reducing STH prevalence in endemic communities in Masbate, the Philippines". This project wanted to evaluate the changes in the knowledge, attitudes and practices of households and children in hygiene and sanitation as well as their STH prevalence rate after undergoing the community-led total sanitation or CLTS process to address the interconnected problem of intestinal parasitic infection and OD practice in the Philippines.

\section{Methodology}

Based on the 2013-15 National Prevalence Survey Report, Region V is on top of the list with highest STH prevalence rate with the Province of Masbate having 77.5\% STH prevalence rate (RITM, 2015). The National Demographic and Health Survey also showed that in Region V, 7\% of households practice OD (PSA \& ICF, 2018). Upon review of existing documents, two (2) highly STH-endemic barangays (Laurente and Dancalan) in San Pascual, Masbate were selected as study sites. The study employed stratified cluster random sampling using households as cluster units. Households were randomly selected and all children aged 2-17 years old were asked to provide stool samples for stool assessment in June 2019. Consenting household heads were interviewed using a pilot-tested questionnaire about the type, ownership, and frequency of use of toilet facility, open defecation practices, water sources, predominant handwashing behavior of their children, including their participation in last year's deworming activities in their community. Children aged 2-17 years old in all participating households were asked to submit stool samples for intestinal parasite screening. During pre-scheduled community assemblies, participants as well as household heads were oriented by trained medical technologists (MdTs) on the purpose of the survey and proper stool collection techniques. Stool samples were submitted by the participants on scheduled days at their barangay health centers $(\mathrm{BHC})$ or to assigned barangay health workers $(\mathrm{BHW})$ that were hired to collect samples. All stool samples were processed using the Kato-Katz (KK) technique (Annex A) on the same day as they were received. Double blinded screening for intestinal parasites using microscopy was employed in all processed stool samples within the week. The laboratory results were given to each household and a copy was given to the municipal health officer (MHO). All children that were positive with any STH infection were prioritized in the next round of deworming activities scheduled in their communities. All data collection forms were double encoded using MS Access before data analysis was done using SPSS 16.0 (SPSS, Inc., 1989-2007). This study used chi-square test of independence to determine the association of variables with the prevalence rate. Households that were not able to provide stool samples were excluded in data analysis.

\section{Results}

The overall stool sample submission rate was $47.83 \%$ from all households interviewed. In the end, only $52.70 \%$ of all interviewed households were included in the analysis. The most cited reasons for non-submission of stool samples were children were reluctant to submit stool samples while some were unable to defecate in the time of the visits. The sex distribution among children who submitted stool samples were roughly equal; $50.7 \%$ male and $49.3 \%$ female. This survey revealed current sanitation status and practices in the selected study sites. 
Table 1

Summary of descriptive statistics

\begin{tabular}{|c|c|c|c|}
\hline & Laurente & Dancalan & Combined \\
\hline \multicolumn{4}{|l|}{ Individual level } \\
\hline Total no. of HHs encoded / interviewed & $108 / 223$ & $126 / 221$ & $234 / 444$ \\
\hline Total no. of stool samples screened / targeted & $276 / 594$ & $310 / 631$ & $586 / 1225$ \\
\hline \multicolumn{4}{|l|}{ Sex (Screened samples) } \\
\hline Male & 139 & 158 & 297 \\
\hline Female & 137 & 152 & 289 \\
\hline \multicolumn{4}{|l|}{ MDA participation in last 12 mos. (No., \%) } \\
\hline Once & 49 & 16 & $65,11.09$ \\
\hline Twice & 191 & 279 & $470,80.20$ \\
\hline More than twice & 19 & 3 & $22,3.75$ \\
\hline Didn't participate in past 12 mos & 17 & 12 & $29,4.95$ \\
\hline \multicolumn{4}{|l|}{ Practicing OD } \\
\hline Yes & $154,56.20$ & $162,52.26$ & $316,53.92$ \\
\hline No & $120,43.80$ & $148,47.80$ & $268,45.73$ \\
\hline \multicolumn{4}{|l|}{ Frequency of $\mathrm{OD}$} \\
\hline Frequent & $85,55.19$ & $102,62.96$ & $187,59.18$ \\
\hline Occasional & $69,44.81$ & $60,37.04$ & $129,40.82$ \\
\hline \multicolumn{4}{|l|}{ Household level (No., \%) } \\
\hline \multicolumn{4}{|l|}{ Toilet type ownership } \\
\hline Water-sealed (w/ septic tank) & $185,67.03$ & $180,58.06$ & $365,62.29$ \\
\hline Water-sealed (w/o septic tank) \& pit latrine & $15,4.84$ & $11,3.99$ & $26,4.44$ \\
\hline None & $80,28.99$ & $115,37.10$ & $195,33.28$ \\
\hline \multicolumn{4}{|l|}{ Source of drinking water } \\
\hline Store-bought water & 38 & 5 & $43,7.34$ \\
\hline Community water system & 76 & 33 & $109,18.60$ \\
\hline Poso & 136 & 120 & $356,60.75$ \\
\hline Dug well & 14 & 51 & $65,11.09$ \\
\hline Collected rainwater & 12 & 1 & $13,2.22$ \\
\hline
\end{tabular}

Majority of screened children (83.95\%) have taken the recommended number of deworming medicine last year (twice or more). Most of them reported that they participated in the MDA conducted at their schools.

Open defecation was found to be a common practice among children. Parents were also asked about how frequent these children defecate in the open, it was found out that more than half of all children screened do it frequently. Parents explained that these children were not yet potty-trained. Others explained that their homes and temporary residences simply lacked toilets (e.g. a child studying in another town may be staying in a relative's house with no toilet facilities). 
Table 1 shows that $33.28 \%$ of households that do not have a decent toilet facility. While the majority of the households (62.92\%) reported that they have septic tanks for their toilets, almost half of the interviewed households do not adhere with the recommended sanitation standards. Aside from that, $27.74 \%$ of those who reported having a toilet facility were sharing it with their relatives living in close proximity to their houses.

Table 1 also describes where households obtain drinking water. The common source of drinking water in these communities was from "poso" or artesian pumping well, which is usually shared by several households.

Figure 2 describes the reported handwashing practices of children. Parents were asked if they observed their children wash their hands before eating, after eating, and after defecation. Majority reported that their children do wash their hands before or after these activities. Children from the younger age groups, who are still under close parental supervision, have their hands personally washed by their parents or guardians. Nearly all the parents surveyed reported soap usage in handwashing, with only a scant few from Brgy. Laurente using only water.

Figure 3 shows the results of a 5-point likert scale with 12 questions designed to measure household heads' knowledge of STH causes, symptoms, and treatment; attitudes; and actual practices against STH infection. Responses to each statement have corresponding points that determined the KAP score for each household. All scores were categorized into three: 'High', 'Average', and 'Low', with a 6-point interval from the lowest possible score. Most participants in both communities scored 'High' on Attituderelated statements while scores on Knowledge- and Practice-related statements veered towards 'Average'. These scores suggest that participants have relatively good knowledge about the illness and they are eager to address STH infections but fall short on actual practice. To illustrate, several parents admitted that while they forbid their children from walking or playing barefoot on soil, they are often disobeyed and cannot always supervise their children.

Table 2 below describes the cumulative prevalence of soil-transmitted helminths (STH) was $41.6 \%$ with moderate to light intensity of infection. Roundworm and whipworm infections were both considerably higher in Brgy. Laurente than Dancalan. On the other hand, Table 4 describes intensity of infection per species in the two study sites. Majority of the Ascaris infections were only light to moderate while Trichuris infections were overwhelmingly light. 
Table 2

STH prevalence rate and intensity of infection per barangay

\begin{tabular}{|llll|}
\hline Intestinal helminth & $\begin{array}{l}\text { Laurente (\%) } \\
\mathbf{n = 2 7 6}\end{array}$ & $\begin{array}{l}\text { Dancalan (\%) } \\
\mathbf{n = 3 1 0}\end{array}$ & $\begin{array}{l}\text { Prevalence (per specie) } \\
\mathbf{n}=\mathbf{5 8 6}\end{array}$ \\
\hline Cumulative STH Prevalence & & $\mathbf{4 1 . 6 \%}$ \\
\hline Roundworm (Ascaris lumbricoides) & $102(36.96 \%)$ & $44(14.19 \%)$ & $146(\mathbf{2 4 . 9 \% )}$ \\
\hline Heavy & $10(9.80 \%)$ & $1(2.27 \%)$ & \\
\hline Moderate & $43(42.16 \%)$ & $20(45.45 \%)$ & \\
\hline Light & $49(48.04 \%)$ & $23(52.27 \%)$ & \\
\hline Whipworm (Trichuris trichiura) & $136(49.28 \%)$ & $26(8.39 \%)$ & $161(\mathbf{2 7 . 4 7 \% )}$ \\
\hline Heavy & $4(2.94 \%)$ & 0 & \\
\hline Moderate & $24(17.64 \%)$ & $1(3.85 \%)$ & \\
\hline Light & $107(78.68 \%)$ & $25(96.15 \%)$ & \\
\hline Hookworm (Ancylostoma duodenale and Necator americanus) & $0(0 \%)$ & $2(0.65 \%)$ & \\
\hline Heavy & 0 & 0 & $8(\mathbf{0 . 3 4})$ \\
\hline Moderate & 0 & $2(100 \%)$ & \\
\hline Light & 0 & 0 & $4(1.29 \%)$ \\
\hline Other (Heterophyids and E. Vermicularis) & $4(1.25 \%)$ & \\
\hline
\end{tabular}

Table 3 describes the variables associated for being tested positive with STH infection among children. From all the variables, only the drinking water source, attitude scores, and participation in deworming activities were found to be related to having STH infection. 
Table 3

Summary of statistical analysis

\begin{tabular}{|ll|}
\hline Variable & $p$ value at $\mathbf{a}=\mathbf{0 . 0 5}$ \\
\hline Household level & \\
\hline Toilet type ownership & 0.295 \\
\hline Drinking water source & $0.002^{\star}$ \\
\hline KAP Score & 0.785 \\
\hline Knowledge Score & 0.340 \\
\hline Attitude Score & $0.022^{\star}$ \\
\hline Practice Score & 0.883 \\
\hline Individual Level & \\
\hline Sex & 0.118 \\
\hline MDA Participation in last 12 mos & $0.025^{\star}$ \\
\hline Practice of OD & 0.873 \\
\hline Frequency of OD & 0.496 \\
\hline Handwashing practice before meal & 0.336 \\
\hline Handwashing practice after meal & 0.955 \\
\hline Handwashing after defecation & 0.407 \\
\hline
\end{tabular}

\section{Discussion}

One-third of the households surveyed still do not have access to decent toilet facilities. Lack of access to toilet facilities have been associated with the lower economic status of the households (WHO/UNICEF,2014). The two barangays belong to a 3rd class municipality with a poverty incidence rate of $57.12 \%$ (PSA, 2016). With this kind of poverty incidence rate, households are expected to prioritize other pressing household needs, compromising with water and sanitation. This might have caused the persistence of OD practice in these barangays. A study conducted in Ghana found out that one of the factors why OD practice persists were the lack of access to physical toilets, among other things (Osumanu, Kosoe, \& Ategeeng, 2019).

Deworming activities at school have been both the preventive and curative intervention for STH infection. Despite being offered for free and known to be effective, achieving the target coverage rate to approximately 19.6 million children in public school alone (WHO, 2016) was daunting for the Integrated Helminth Control Program of the DoH. In this study, $95.04 \%$ of the screened samples reported that they received deworming drugs in the last 12 months, however, only $83.95 \%$ participated in the recommended number of deworming intake. This is still below the national target for MDA coverage of at least $85 \%$, wherein positive impact on reducing the burden of the disease is expected (Department of Health, 2015). This results on MDA participation was consistent with the national status of deworming coverage. In WHO reported data, the national coverage for deworming was 75.7\% (Lo, et al, 2020). But unlike in sanitation, it is not the lack of access to free and safe medication that poses a problem. It is the lack of confidence of the parents to the teachers' ability to take care of their children whenever adverse reactions to medications might occur during the deworming day (Lorenzo, Manzanilla, Cortel, \& Tangog, 2019; Sinha, et al, 2011).

The KAP scores shown in this study suggest that the community is very aware of how the disease is transmitted and its symptoms, and they have a good attitude towards the disease prevention. However, they fell short on practices. Health workers played an important role in advocacy efforts on WASH and STH illness. Studies revealed that community health workers were the main source of information in these communities (Parikh, et. al., 2013; Lorenzo, et al 2019). Parents were aware that their children sometimes walk barefooted on soil especially during playtime, picking up and eating dropped foods from the bare ground, and 
even nail biting. Although these practices were discouraged, disobedience and not being with their children most of the time led to continued practice.

This study also found out that Trichuris trichiura and Ascaris lumbricoides accounted for $27.47 \%$ and $24.9 \%$ of STH infections in both sites, respectively, with the majority being light intensity infections. Other surveys conducted in other provinces in the Philippines also revealed a similar trend of prominence of $T$. trichiura infection followed by A.lumbricoides and hookworms (Mationg, et al, 2017; Ross et al, 2017; Soares, Salamat, Leonardo, Gray, Carabin, Halton, et al. 2015). The communities with lack of access to proper water, hygiene, and sanitation facilities were usually found to have high STH prevalence rates (Pullan, et. al, 2014; Ross et al, 2017). This lack of access to sanitation facilities exacerbates the practice of OD which allows the continued risk of STH transmission (Ziegelbauer,et. al., 2012). Studies found that human excreta from STH infected individuals can contaminate soil which can be one of the modes of transmission for STH infections among school aged children (Nery, et. al., 2019). A survey on soil from farms from North and South Luzon also revealed that they harbor these intestinal parasites, and those farms that utilized manure as fertilizer have a higher rate of parasites seen in soil samples compared to those that use chemical fertilizers (Paller \& Babia-Abion, 2019). This study suggested that the presence of parasites in soil only suggest unhygienic practices like open defecation.

The continued practice of OD due to lack of access to improved sanitation facilities can also contaminate water sources intestinal parasites which can be transferred to human thru contact. The most common source of drinking water in the selected communities were poso or artesian pumping wells, which oftentimes not treated. A study conducted in Cebu found out that improved water supply and environmental sanitation practices reduced the risk of reported diarrhea in clinics (Baltazar, Briscoe, Mesola, et al., 1988). A systematic review of 94 studies was conducted that found out that the use of treated water and piped water access reduce the risk of STH infections (Strunz, Addiss, Stocks, Ogden, Utzinger \& Freeman, 2014). While STH is known to be transmitted thru soil and not water, the possibility of drinking untreated water contaminated soil harboring these intestinal parasites can pose risk and has to be explored further.

Among different approaches, CLTS was found to be popular in many countries in Asia and Africa as a low input, low cost, and community-initiated strategy to address hygiene and sanitation (Kar, 2005). While taunted to be participatory and sustainable in terms of implementation, there is very little interest in looking at its impact on public health and sanitation. In fact, there is only one study in the country that looked into the possible effects of CLTS in STH prevalence. It revealed that CLTS communities have relatively higher STH prevalence rate compared with non-CLTS communities in Southern Leyte (Belizario, et al, 2015). The main reason to explain this was the reversion to OD due to lack of monitoring after ZOD certification. This was also one of the challenges reported in East Asia coupled with lack of commitment to sustain among government agencies (UNICEF EAPRO, 2015). An archipelagic country like the Philippines presents geographic and spatial differences per region, highlights the necessity of providing improved sanitation facilities and behavior change interventions to supplement MDA for STH control and prevention (Soares, et al, 2015). Like hitting two birds in one stone, CLTS is one of the approaches that can be explored and strengthened to address not only sanitation challenges among rural communities but also public health issues like STH infections among children.

\section{Conclusion}

The persistence of intestinal parasitic infection among school aged children was caused by a myriad of factors. Lack of access to decent toilet facilities due to socio-economic factors have led people to practice open defecation. Open defecation can contaminate drinking water sources which can lead to public health concerns if left unaddressed. Parent's lack of confidence in teachers' ability to handle and address adverse reactions to deworming drugs led to an unmet MDA coverage target.

While this study did not conclude association of OD practice and STH infection among children, it has described the sanitation and prevailing KAP in STH endemic communities like San Pascual, Masbate. This is the preliminary data for a bigger study that would want to see the effects of CLTS in decreasing the STH prevalence in target communities but also changing their hygiene and sanitation practices. 
This study provided evidence to strengthen strategies that not only provide disease-specific response but also change the attitudes and practices of the target communities. While MDA is proven to be a cost-effective strategy for STH infections among school aged children, it cannot prevent reinfection unless hygiene and sanitation are improved simultaneously. There is a need to strengthen support and implementation of strategies like community-led total sanitation or CLTS can provide sustainable hardware and software solutions to hygiene and sanitation. Without the partnership and commitment of the local government agencies and its people, achieving and sustaining target MDA coverage and ZOD status will be daunting and intestinal parasitic infection among children will continue to persist. For a developing country like the Philippines, a strategy that can address two of the most persisting problems in rural communities is something that is worth exploring, and CLTS is one of them.

\section{STUDY LIMITATIONS}

One of the limitations of the study was the potential biased on self-reported information and observed behavior among children. Researchers relied on probing skills of trained data collection team and sincerity of the respondents' providing the right information. Adopting alternative ways of recording accurate information on practices and behavior can provide more information between the link of intestinal parasitic infections and OD practice among children. Information about the possible effects of CLTS in improving sanitation practices and preventing transmission of STH has yet to be proved in this paper. A follow up survey was intended after one of the communities has undergone CLTS activities and has declared a ZOD community. This will prove the need of integrating strategies to address different health and sanitation outcomes. Moreover, stakeholder and community engagement are necessary to ensure that interventions like CLTS are implemented so there are opportunities to study the implementation process.

\section{Declarations}

\section{ETHICAL APPROVAL AND CONSENT TO PARTICIPATE}

The study was thoroughly reviewed and was given an approval by Institutional Review Board of Research Institute for Tropical Medicine in May 24, 2019.

\section{CONSENT FOR PUBLICATION}

The authors consent to the publication of the manuscript to Infectious Diseases of Poverty, should the article be accepted by the Editor-in-chief upon completion of the refereeing process.

\section{AVAILABILITY OF DATA AND MATERIALS - N/A}

\section{COMPETING INTERESTS}

Authors declared that there are no competing interests in the publication of this manuscript.

\section{FUNDING}

This work is funded by the World Health Organization Special Programme for Research and Training in Tropical Diseases (WHOTDR) thru the Joint TDR/WPR Small Grant Scheme for Implementation Research in Infectious Disease of Poverty (Project ID: 2019.4.PHL.1 .MVP). The funders had no role in study design, data collection and analysis, decision to publish, or preparation of the manuscript.

\section{AUTHORS' INFORMATION}

Pauline Joy Lorenzo, Charmaine Joy Berte, Dazzle Kane Cortel, Duane Raphael Manzanilla, Christian Anthony Luna, Jennifer Luchavez, and Winston Palasi contributed equally to this work.

\section{Contributions}


All authors were involved in project conceptualization and design. PJL, DKC, DRM, and CJB conducted data collection and data analysis. JSL provided supervision and logistical support during the data collection phase. PJL, DKC, DRM, CJB, and CAL draft manuscript. JSL and WAP provided technical inputs for final manuscript.

$\underline{\text { Affiliations }}$

Research Institute for Tropical Medicine, 9002 Research Drive, Filinvest Corporate City, Alabang, Muntinlupa City, Metro Manila, Philippines 1781

Pauline Joy Lorenzo, Charmaine Joy Berte, Dazzle Kane Cortel, Duane Raphael Manzanilla, Christian Anthony Luna, and Jennifer Luchavez

Department of Health, Disease Prevention and Control Bureau, San Lazaro Compound, Sta. Cruz, Manila, Philippines

Winston Palasi, MD

Philippine Institute for Development Studies

18F Three Cyberpod Centris - North Tower, EDSA cor. Quezon Ave., Quezon City, Metro Manila, Philippines

Pauline Joy Lorenzo

\section{ACKNOWLEDGEMENTS}

We thank the Department of Parasitology for their off- and on-site logistical support. Our sincerest gratitude to Ralph Reyes and Catherine Montano for their invaluable contribution in the processing and screening stool samples and assisting during household surveys. We would also like to thank all the Local Government Unit of San Pascual and the Municipal Health Office with their support and assistance in coordinating with the households and all the barangay health workers who assisted us in gathering and collecting stool samples from households. Most importantly, we would like to thank the people of San Pascual, Masbate for welcoming and participating in the research activities.

\section{References}

Anderson, R.M., Truscott, J.E., Pullan, R.L., Brooker, S.J., Hollingsworth, T.D. 2013. "How effective is school-based deworming for the community-wide control of soil-transmitted helminths?" PLoS Negl Trop Dis 7: e2027.

Baltzar, J., Briscoe, J., Mesola, V., Moe, C., Solon, F., Vanderslice, J., \& Young, B. 1988. “Can case-control method be used to assess the impact of water supply and sanitation on diarrhoea?" Bulletin of the World Health Organization, 66;5;627-635. DOI:

https://www.ncbi.nlm.nih.gov/pmc/articles/PMC2491179/?page=1

Belizario, V.Y., Liwanag, H.J., Naig, J.R., Chua, P.L., Madamba, M.I., Dahildahil, R.O. 2015. “Parasitological and nutritional status of school-age and preschool-age children in four villages in Southern Leyte, Philippines: Lessons for monitoring the outcome of Community-Led Total Sanitation." Acta Tropica, Volume 141, Part A, Pages 16-24, ISSN 0001-706X.

https://doi.org/10.1016/j.actatropica.2014.09.008.

Belizario, V.Y., Tuliao, A.H., Totanes, F.G., \& Asuncion, C.L. 2013. "Optimizing school-based intestinal helminth control interventions in the Philippines". Pediatric Infectious Disease Society of the Philippines Journal 14:1; 2-13.

Clements A.C., Bosque-Oliva E, Sacko M., Landouré, A., Dembélé, R., et al. 2009. "A comparative study of the spatial distribution of schistosomiasis in Mali in 1984-1989 and 2004-2006." PLoS Negl Trop Dis 3: e431.

Department of Health. 2015. "Guidelines on the Implementation of the National School Deworming Day" Administrative Order No. 2015-0030. San Lazaro Compound, Sta. Cruz, Manila, Philippines.

Doenhoff, M.J., Cioli, D., \& Utzinger, J. 2009. “Praziquantel: mechanisms of action, resistance and new 
derivatives for schistosomiasis." Curr Opin Infect Dis 21: 659-67.

Kar, K. 2005. Practical guide to triggering community-led total sanitation (CLTS). Institute of Development Studies. University of Sussex, Brighton, United Kingdom. ISBN 185864884 X Retrieved from:

https://www.communityledtotalsanitation.org/sites/communityledtotalsanitation.org/files/Guidelines_for_triggering_CLTS_0.pdf

Lo, N. C., Gupta, R., Addiss, D. G., Bendavid, E., Heft-Neal, S., Mikhailov, A., ... \& Mbabazi, P. S. (2020). "Comparison of World Health Organization and Demographic and Health Surveys data to estimate sub-national deworming coverage in pre-school aged children." PLoS neglected tropical diseases, 14(8), e0008551.

Lorenzo, P.J., Manzanilla, D.R., Cortel, D.K. \& Tangog, E. 2019. "Community perceptions of mass drug administration for soiltransmitted helminthiasis and schistosomiasis in selected schools in the Philippines." Infect Dis Poverty 8 , 87. https://doi.org/10.1186/s40249-019-0595-8

Mationg, M. L. S., Gordon, C. A., Tallo, V. L., Olveda, R. M., Alday, P. P., Reñosa, M. D. C., ... \& Halton, K. 2017. “Status of soiltransmitted helminth infections in schoolchildren in Laguna Province, the Philippines: Determined by parasitological and molecular diagnostic techniques." PLoS neglected tropical diseases, 11(11), e0006022.

Nery, S. V., Pickering, A. J., Abate, E., Asmare, A., Barrett, L., Benjamin-Chung, J., ... \& Ercumen, A. (2019). "The role of water, sanitation and hygiene interventions in reducing soil-transmitted helminths: interpreting the evidence and identifying next steps." Parasites \& vectors, 12(1), 273.

Njuguna, J. 2016. "Effect of eliminating open defecation on diarrhoeal morbidity: an ecological study of Nyando and Nambale sub-counties, Kenya". BMC Public Health 16, 712. https://doi.org/10.1186/s12889-016-3421-2

Paller, V.G.V., \& Babia-Abion, S. 2019. "Soil-transmitted helminth (STH) eggs contaminating soils in selected organic and conventional farms in the Philippines." Parasite Epidemiology and Control 7;

e00119. DOI: https://doi.org/10.1016/j.parepi.2019.e00119

Parikh, D. S., Totañes, F. I., Tuliao, A. H., Ciro, R. N., Macatangay, B. J., \& Belizario, V. Y. (2013). “Knowledge, attitudes and practices among parents and teachers about soil-transmitted helminthiasis control programs for school children in Guimaras, Philippines." Southeast Asian J Trop Med Public Health, 44(5), 744-52.

Philippine Statistics Authority (PSA). 2016. 2012 Municipal and City Level Poverty Estimates. Quezon City, Philippines. Retrieved December 1, 2020, from https://psa.gov.ph/psa-press-release-tags/poverty-small-area-estimate

Philippine Statistics Authority (PSA) \& ICF. 2018. Philippines National Demographic and Health Survey 2017. Quezon City, Philippines \& Rockville, Maryland, USA: PSA and ICF.

Pickering, A.J., Djebbari, H., Lopez, C., Coulibaly, M., \& Alzua, M.L. 2015. “Effect of a community-led sanitation intervention on child diarrhoea and child growth in rural Mali: a cluster-randomised controlled trial." The Lancet Global Health,3; 11, Pages e701e711,ISSN 2214-109X, https://doi.org/10.1016/S2214-109X(15)00144-8

Prüss-Ustün, A., Bartram, J., Clasen, T., Colford, J.M. Jr, Cumming, O., Curtis, V., Bonjour, S., et al. 2014. “Burden of disease from inadequate water, sanitation and hygiene in low- and middle-income settings: a retrospective analysis of data from 145 countries." Trop Med Int Health. 2014 19(8):894-905. DOI: 10.1111/tmi.12329

Pullan, R. L., Smith, J. L., Jasrasaria, R., \& Brooker, S. J. (2014). “Global numbers of infection and disease burden of soil transmitted helminth infections in 2010". Parasites \& vectors, 7(1), 37.

Research Institute for Tropical Medicine (RITM). 2015. National survey on the prevalence of Soil-transmitted helminths (STH), Schistosomiasis, and other intestinal parasitic infections among public school children (day-care, elementary, and high school) in the Philippines. Muntinlupa City, Manila. 
Ross A.G.P., Bartley, P.B., Sleigh, A.C., Olds, G.R., Li, Y., et al. 2002. “Schistosomiasis.” N Eng J Med 346: 1212-19.

Ross, A.G.P., Olveda, R.M., McManus, D.P., Harn, D.A., Chy, D., Li, Y., Tallo, V., \& Ng, S. 2017. “Risk factors for human helminthiases in rural Philippines". International Journal of Infectious Diseases; 54; Page 150-155 DOI:

https://doi.org/10.1016/j.ijid.2016.09.025

Sinha, D., Totanes, F.I.G., Tuliao, A., Ciro, R.N., Macatangay, B.J.C., \& Belizario, V.Y. 2011. "Factors to consider for the elimination of soil-transmitted helminthiasis in school children." Poster Presentation. IDSA 49th Annual Meeting. Boston, USA. URL:

https://idsa.confex.com/idsa/2011/webprogram/Paper30978.html

Soares Magalhães R.J., Salamat, M.S., Leonardo, L., Gray, D.J., Carabin, H., Halton, K., et al. 2015 “Mapping the Risk of SoilTransmitted Helminthic Infections in the Philippines." PLoS Negl Trop Dis 9(9): e0003915.

DOI: https://doi.org/10.1371/journal.pntd.0003915

Strunz, E. C., Addiss, D. G., Stocks, M. E., Ogden, S., Utzinger, J., \& Freeman, M. C. (2014). Water, sanitation, hygiene, and soiltransmitted helminth infection: a systematic review and meta-analysis. PLoS Med, 11(3), e1001620.

UNICEF East Asia and Pacific Regional Office. 2015. Second review of community-led total sanitation in East Asia and Pacific: Regional Report. UNICEF EAPRO. Bangkok, Thailand.

WHO/UNICEF Joint Monitoring Programme for Water Supply and Sanitation. 2012. Progress on Drinking Water and Sanitation: 2012 Update, World Health Organization: Geneva, Switzerland, p. 60.

WHO/UNICEF Joint Monitoring Program. 2015. Progress on Sanitation and Drinking Water: 2014 Update, WHO/UNICEF, New York, NY, USA.

WHO, UNICEF, 2017. Progress on Drinking Water, Sanitation and Hygiene: 2017 Update

and SDG Baselines. World Health Organization. Geneva, Switzerland.

Zuin, V., Delaire, C., Peletz, R., Cock-Esteb, A., Khush, R., \& Albert, J. 2019. "Policy Diffusion in the Rural Sanitation Sector: Lessons from Community-Led Total Sanitation (CLTS)". World Development, 124, 104643, ISSN 0305-750X.

https://doi.org/10.1016/j.worlddev.2019.104643

\section{Figures}




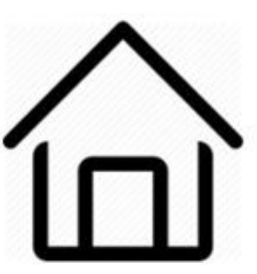<smiles>[GeH3]</smiles>

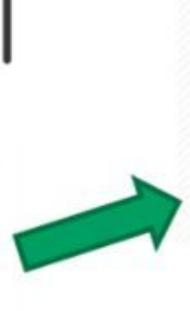

$\checkmark$ Stool samples will be submitted to rotating $\mathrm{BHWs}$ or submitted to $\mathrm{BHCs}$ on scheduled date

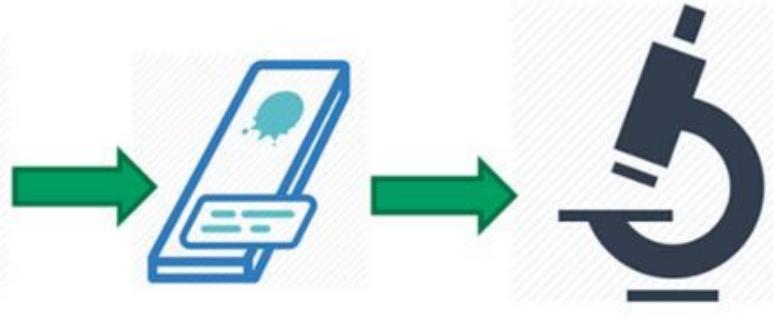

$\checkmark$ MdTs will prepare stools for $\mathrm{KK}$ technique ( $\leq 4 \mathrm{hrs})$
$\checkmark$ Double blinded diagnosis (MdT1 and MdT2) ( $\leq 24 \mathrm{hrs})$
$\checkmark$ Validation (MdT3) ( $\leq 7$ days)

Figure 1

Data collection process flow

\section{Children's handwashing practices}

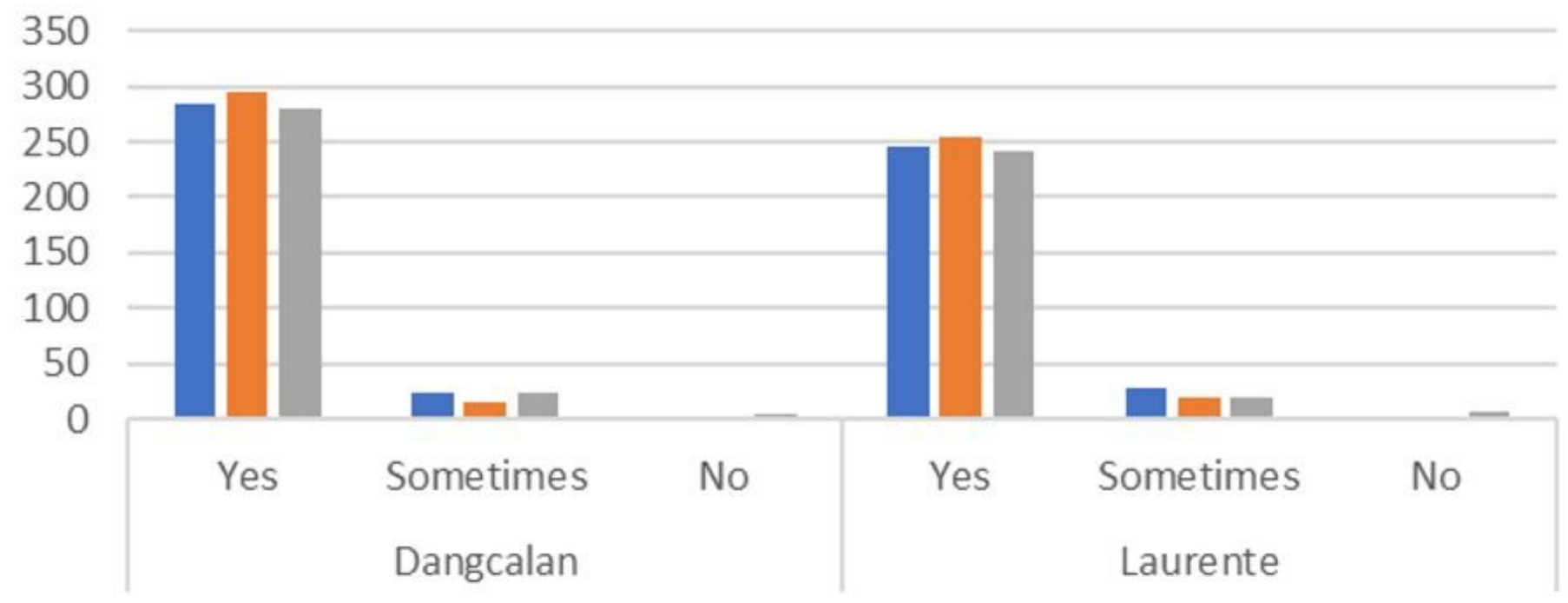

- Before meals after meals $\quad$ After defecating

Figure 2

Reported handwashing practice among children 


\begin{tabular}{|c|c|c|c|c|c|c|c|c|}
\hline \multicolumn{9}{|l|}{$100 \%$} \\
\hline \multicolumn{9}{|l|}{$90 \%$} \\
\hline \multicolumn{9}{|l|}{$80 \%$} \\
\hline \multicolumn{9}{|l|}{$\begin{array}{l}70 \% \\
60 \%\end{array}$} \\
\hline \multirow{2}{*}{\multicolumn{9}{|c|}{$50 \%$}} \\
\hline \multirow{2}{*}{\multicolumn{8}{|c|}{$40 \%$}} & \\
\hline \multirow{2}{*}{\multicolumn{9}{|c|}{$30 \%$}} \\
\hline & & & & & & & & \\
\hline \multirow{2}{*}{\multicolumn{9}{|c|}{$10 \%$}} \\
\hline & & & & & & & & \\
\hline \multirow[t]{2}{*}{$0 \%$} & Knowledge & Attitude & Practice & Over-all KAP & Knowledge & Attitude & Practice & Over-all KAP \\
\hline & \multicolumn{4}{|c|}{ Laurente } & \multicolumn{4}{|c|}{ Dancalan } \\
\hline Low & 3 & 1 & 9 & 0 & 0 & 0 & 17 & 0 \\
\hline Average & 222 & 11 & 161 & 37 & 232 & 2 & 193 & 62 \\
\hline - High & 51 & 264 & 106 & 239 & 78 & 308 & 100 & 248 \\
\hline \multicolumn{9}{|c|}{ घHigh $\mathbf{n}$ Average $=$ Low } \\
\hline
\end{tabular}

\section{Figure 3}

Household KAP score range 